\title{
PREVALENCIA DE DIENTES SUPERNUMERARIOS EN NIÑOS DE 6 A 12 AÑOS EN LA CIUDAD DE HUÁNUCO DEL 2012 AL 2016
}

\author{
PREVALENCE OF SUPERNUMERARY TEETH IN CHILDREN FROM 6 TO 12 YEARS OLD \\ IN THE CITY OF HUÁNUCO FROM 2012 TO 2016 \\ Milagros Zevallos Melgar ${ }^{1}$, Carol Magaly Cárdenas-Flores ${ }^{1}$
}

\section{RESUMEN}

Objetivo: Determinar la prevalencia de dientes supernumerarios identificados mediante radiografías panorámicas en niños de 6 a 12 años de la ciudad de Huánuco, durante el período 2012-2016. Materiales y métodos: La muestra fue de 523 radiografías panorámicas de pacientes entre 6 y 12 años. Se realizó la observación detallada de cada radiografía panorámica por sextantes verificando cada una de las piezas dentarias y gérmenes dentarios adyacentes. Se usó una ficha para el registro de la información donde se indicó la edad del paciente y el tipo de alteración en el número de piezas dentarias con sus respectivos indicadores: ubicación, anatomía por sextantes y tipo de pieza dentaria afectada. Resultados: La prevalencia de dientes supernumerarios en niños de 6 a 12 años es del 12,81\% encontradas en 67 radiografías panorámicas, los cuales se encontraron en la arcada superior un $67,16 \%$ y en el inferior, un $32,83 \%$. En cuanto a la ubicación el mesiodens representa el $47,76 \%$ y el parapremolar, un $25,37 \%$. De acuerdo con el sexo, tenemos un porcentaje del 52,23\% para el sexo masculino y del $47,76 \%$ para el sexo femenino. Conclusión: La prevalencia de dientes supernumerarios en niños de 6 a 12 años en la ciudad de Huánuco es del 12,81\%. La ubicación más frecuente fue el mesiodens. De acuerdo con la morfología, el tipo más frecuente es el dismórfico.

Palabras clave: anomalías dentarias, radiografía panorámica, diente supernumerario, prevalencia

\section{ABSTRACT}

Objective: To determine the prevalence of supernumerary teeth identified by panoramic radiography in children from 6 to 12 years of age in the city of Huánuco during the period 2012-2016. Materials and Methods: The sample consisted of 523 panoramic radiographs of patients aged from 6 to 12 years. Detailed

Citar como: Zevallos M, Cárdenas C. Prevalencia de dientes supernumerarios en niños de 6 a 12 años en la ciudad de Huánuco del 2012 al 2016. Rev Cient Odontol (Lima). 2018; 6 (2): 158-166.

1 Universidad Científica del Sur. Lima, Perú. 
observation of each panoramic radiograph was conducted for each sextant, verifying each tooth and adjacent tooth bud. A card was used to record the information regarding the patient's age and the type of alteration in the number of teeth and their respective indicators: location, anatomy of each sextant and type of tooth affected. Results: The prevalence of supernumerary teeth in children from 6 to 12 years of age was $12.81 \%$, based upon 67 panoramic radiographs, $67.16 \%$ of which were located in the upper arch and $32.83 \%$ in the lower arch. In terms of location, $47.76 \%$ were mesiodens, and $25.37 \%$ were parapremolar. In terms of gender, the percentage in males was $52.23 \%$, and in females $47.76 \%$. Conclusion: The prevalence of supernumerary teeth in children from 6 to 12 years of age in the city of Huánuco was $12.81 \%$. The most frequent location was the mesiodens, while the most frequently occurring form was dysmorphic.

Keywords: Dental anomalies, panoramic radiography, supernumerary teeth, prevalence.

\section{INTRODUCCIÓN}

La región cráneo-maxilofacial es parte del sistema estomatognático. Si nos abocamos a una parte de la región oral y específicamente a las alteraciones que ocurren en la formación de los órganos dentarios, podemos encontrar anomalías como odontomas, agenesias dentarias, dientes supernumerarios y otros tipos más (1).

Los dientes supernumerarios se definen como dientes en exceso sobre la fórmula dentaria normal, ya sea mayor a 22 dientes en la dentición decidua o mayor a 32 piezas en la dentición permanente (2). Se presencia se debe a alteraciones en las fases de inicio y proliferación (3). Las causas todavía son desconocidas, pero se han planteado varias teorías para explicarlas como una anomalía asociada a síndromes o a un origen multifactorial en el cual intervienen factores genéticos y ambientales (4).

En cuanto a su etiología embriológica, tenemos dos teorías muy aceptadas. Una es la teoría de la hiperactividad de la lámina dental, la cual nos dice que, durante la evolución del germen dentario, la lámina dental degenera y da lugar a la persistencia de remanentes; si estos restos epiteliales son influenciados por factores inductivos, se formará un germen dental en exceso o un odontoma (5). La otra teoría es la dicotomía del germen dental, la cual afirma que en el estadio de brote o yema el diente se divide en dos partes iguales o de diferentes tamaños, lo que da como resultado dos dientes iguales o un diente de tamaño normal y otro anormal, siendo más pequeño o rudimentario, producto de infecciones o traumatismos en este estadio $(6,7)$.

Los dientes supernumerarios, de acuerdo con su morfología, pueden ser eumórficos, iguales al diente adyacente (8), o dismórficos, que a su vez pueden ser cónicos tuberculados o molariformes (9). Según su ubicación, tenemos los mesiodens, que son los ubicados en la línea media entre los incisivos centrales superiores, y los que con más frecuencia se presentan, además de los paparemolares, distomolares, paramolares y otros (10).

El diagnóstico temprano y el tratamiento oportuno nos ayudarán a evitar múltiples complicaciones que pueden presentarse por esta anomalía (11).

Nuestra investigación se realizó en niños de 6 a 12 años que residen en la ciudad de Huánuco, entre los años 2012 y 2016. No se encontraron estudios epidemiológicos anteriores sobre las alteraciones dentarias en número de esta ciudad, 
por lo que resulta de interés observar su frecuencia.

\section{MATERIALES Y MÉTODOS}

Se realizó un estudio observacional descriptivo de radiografías panorámicas en pacientes pediátricos, de edades comprendidas entre 6 y 12 años, que fueron tomadas en el centro radiológico CERO de la ciudad de Huánuco, en un período de cinco años (enero de 2012 a diciembre de 2016).

Cada radiografía panorámica incluida en el estudio fue evaluada por el investigador en una laptop con procesador Corel i7 y con la ayuda de una lupa. El análisis se realizó en los ambientes del centro radiológico CERO.

Las observaciones consistieron en la revisión y análisis detallado de cada radiografía panorámica por sextantes, a partir del sextante superior derecho y luego del anterosuperior, superior izquierdo, inferior izquierdo, anterior inferior e inferior derecho, verificando cada una de las piezas dentarias y gérmenes dentarios adyacentes.

Se consideró diente supernumerario cuando se apreció radiográficamente una pieza o un germen dentario que se encontrase en exceso con respecto a la fórmula dentaria normal (figuras 1, 2, 3 y 4).

Se usó una ficha para el registro de la información recolectada durante la fase de investigación, en la que se indicará la edad del paciente y el tipo de alteración de piezas dentarias con sus respectivos indicadores: número de piezas afectadas, ubicación, anatomía por sextantes y tipo de pieza dentaria afectada.

Para evaluar la prevalencia de dientes supernumerarios, su presencia, frecuencia y ubicación, así como el género y la edad de los pacientes, se elaboraron tablas de frecuencia simple y de contingencia. También se utilizó la prueba de chi cuadrado $\left(X^{2}\right)$ para establecer si las diferencias que se encontraron fueron características de la población o solo se debieron al azar. Se consideró una diferencia significativa cuando existió más del $95 \%$ de confianza $(p<0,05)$.

Se procesaron los datos mediante el uso del paquete estadístico SPSS 22.0.

\section{RESULTADOS}

Se analizaron 523 radiografías panorámicas de pacientes pediátricos en dentición mixta de 6 a 12 años.

La prevalencia de dientes supernumerarios se presenta en la tabla 1 , donde se observa que existe una prevalencia del $12,81 \%$ (67) de presencia de dientes supernumerarios y un $87,19 \%$ (256) de ausencia.

Según la ubicación de los dientes supernumerarios, en la tabla 2 , se observa que el $47,76 \%$ (32) tienen ubicación mesiodens, el 25,37\% (17) corresponden al parapremolar, el 19,41\% (13) pertenecen

Tabla 1. Prevalencia de dientes Supernumerarios Registrada en Radiografías Panorámicas EN NIÑOS DE 6 A 12 AÑOS EN LA CIUDAD DE HUÁNUCO, DE 2012 A 2016

\begin{tabular}{|l|c|c|}
\hline Dientes supernumerarios & $\mathrm{n}^{\circ}$ & $\%$ \\
\hline No & 456 & 87,19 \\
\hline Sí & 67 & 12,81 \\
\hline Total & 523 & 100,0 \\
\hline
\end{tabular}




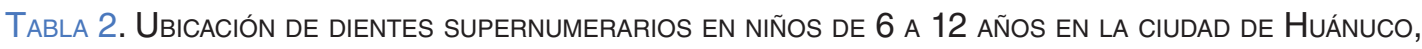
DE 2012 A 2016

\begin{tabular}{|l|c|c|}
\hline Ubicación & n. ${ }^{\circ}$ & $\%$ \\
\hline Mesiodens & 32 & 47,76 \\
\hline Parapremolar & 17 & 25,37 \\
\hline Otro & 13 & 19,41 \\
\hline Distomolar & 3 & 4,48 \\
\hline Paramolar & 2 & 2,98 \\
\hline Total & 67 & 100,0 \\
\hline
\end{tabular}

Tabla 3. Morfología de dientes supernumerarios en NIÑOS de 6 a 12 años en LA CIUDAD dE HUÁNUCO, DE 2012 A 2016

\begin{tabular}{|l|c|c|}
\hline Morfología & $\mathrm{n} \cdot{ }^{\circ}$ & $\%$ \\
\hline Dismórfico & 38 & 56,71 \\
\hline Eumórfico & 29 & 43,29 \\
\hline Total & 67 & 100,0 \\
\hline
\end{tabular}

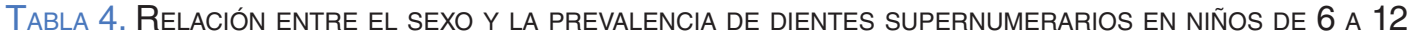
AÑOS EN LA CIUDAD DE HUÁNUCO, DE 2012 A 2016

\begin{tabular}{|l|c|c|c|c|c|}
\multirow{2}{*}{ Sexo } & \multicolumn{5}{|c|}{ SEXO * N. ${ }^{\circ}$ DSN TABULACIÓN CRUZADA } \\
\cline { 2 - 6 } & & DOS & UNO & TOTAL & PSN \\
\hline Masculino & 203 & 4 & 31 & 238 & 0,059 \\
\hline Femenino & 253 & 3 & 29 & 285 & \\
\hline Total & 456 & 7 & 60 & 523 & \\
\hline
\end{tabular}

Prueba estadística Chi cuadrado $=12,145$

TABLA 5. ReLACIÓN ENTRE LA EDAD Y LA PREVALENCIA DE DIENTES SUPERNUMERARIOS EN NIÑOS DE 6 A 12 AÑOS EN LA CIUDAD DE HUÁNUCO, DE 2012 A 2016

\begin{tabular}{|l|l|c|}
\hline Edad & $n \cdot{ }^{\circ}$ & $\%$ \\
\hline $6-9$ años & 33 & 49,25 \\
\hline $10-12$ años & 34 & 50,75 \\
\hline Total & 67 & 100,0 \\
\hline
\end{tabular}

a otro tipo de diente supernumerario (entre incisivo lateral y canino), el 4,48\% (3) tienen ubicación distomolar, y el 2,98\%

(2) presenta una ubicación paramolar.

De acuerdo con la morfología de los dientes supernumerarios, vemos en la tabla 3 que el $56,71 \%$ (38) es dismórfico y el 43,29\% (29) eumórfico.
En la tabla 4 presentamos la relación entre el sexo y la prevalencia de dientes supernumerarios en niños de 6 a 12 años en la ciudad de Huánuco, de 2012 a 2016. Como se aprecia, la relación entre el sexo y la presencia de dientes supernumerarios no es significativa.

La tabla 5 presenta la relación entre la edad y la prevalencia de dientes 
supernumerarios en niños de 6 a 12 años en la ciudad de Huánuco, de 2012 a 2016.

\section{DISCUSIÓN}

En el estudio se fueron utilizadas radiografías panorámicas como instrumento para el diagnóstico de las anomalías de número.

Sánchez (12) determinó la frecuencia y distribución de dientes supernumerarios no asociados a síndromes en dientes permanentes en una muestra chilena. Al compararlo con nuestro estudio, encontramos similitudes en los resultados como una mayor proporción en el género masculino y frecuencia de supernumerario al mesiodens. De acuerdo con la forma, el supernumerario dismórfico obtuvo el mayor porcentaje, con un $68 \%$, y fue igualmente mayor en nuestro estudio. Por otra parte, también en la orientación vertical se ve una similitud en el estudio de un $68 \%$ parecido al que obtuvimos en nuestro estudio, con un porcentaje del $85,02 \%$. Para finalizar, se encontró en ambos estudios una mayor predilección por el maxilar que por la mandíbula.

En el estudio de Jammal y Silva (13) se determina la prevalencia y la ubicación de los dientes supernumerarios no asociados a síndromes. Aquí se encontró un porcentaje de dientes supernumerarios del $55 \%$ para el sexo masculino y el $45 \%$ para el sexo femenino, similar al de nuestro estudio, ya que en este se encontró un porcentaje del $52,23 \%$ para el sexo masculino y del $47,76 \%$ para el sexo femenino. También podemos comparar los porcentajes de mesiodens que en el estudio citado arroja un 35\% y en el nuestro alcanza un porcentaje del $47,76 \%$. Asimismo, el porcentaje de paramolar fue del $22,5 \%$ y en nuestro estudio solo alcanzó un $2,98 \%$; por otro lado, el porcentaje de caninos e incisivos laterales suplementarios fue del $10 \%$ y en nuestro estudio estos supernumerarios llegaron al $19,40 \%$. Por tanto, de ambos estudios se puede concluir que el supernumerario más común es el mesiodens $y$, por ende, el maxilar es donde resulta más frecuente su presencia.

La investigación de Acosta (14) determinó cuáles fueron las anomalías de número en radiografías panorámicas de niños de 6 a 12 años en la población arequipeña. El hallazgo más importante fue la comparación para conocer si existe una relación estadística entre la hiperodoncia y el sexo masculino, siendo este el que con mayor probabilidad presenta dicha anomalía. En nuestro estudio obtuvimos los mismos resultados, así como que el mesiodens es el que muestra mayor porcentaje de presencia de dientes supernumerarios.

Por último, Serrano (15) realizó una investigación para determinar la frecuencia de anomalías dentarias de número en niños de 8 a 12 años en la provincia de Chiclayo, en el período 2012-2013. En dicho estudio observamos que lo más prevalente entre agenesias y supernumerarios fueron las agenesias de mayor porcentaje, pero en cuanto a los dientes supernumerarios nos refiere que, en su mayoría, se encontraron en pacientes del sexo masculino, con lo que coincide nuestro estudio.

Contribución del autor: Milagros Zevallos Melgar y Carol Magaly Cárdenas Flores han participado en la concepción del artículo, la recolección de datos, su redacción y aprobación de la versión final.

Fuente de financiamiento: Autofinanciada. 
Conflictos de interés: Las autoras declaran no tener conflictos de interés de ningún tipo.

\section{RADIOGRAFÍAS PANORÁMICAS DONDE SE OBSERVAN DIENTES SUPERNUMERARIOS}

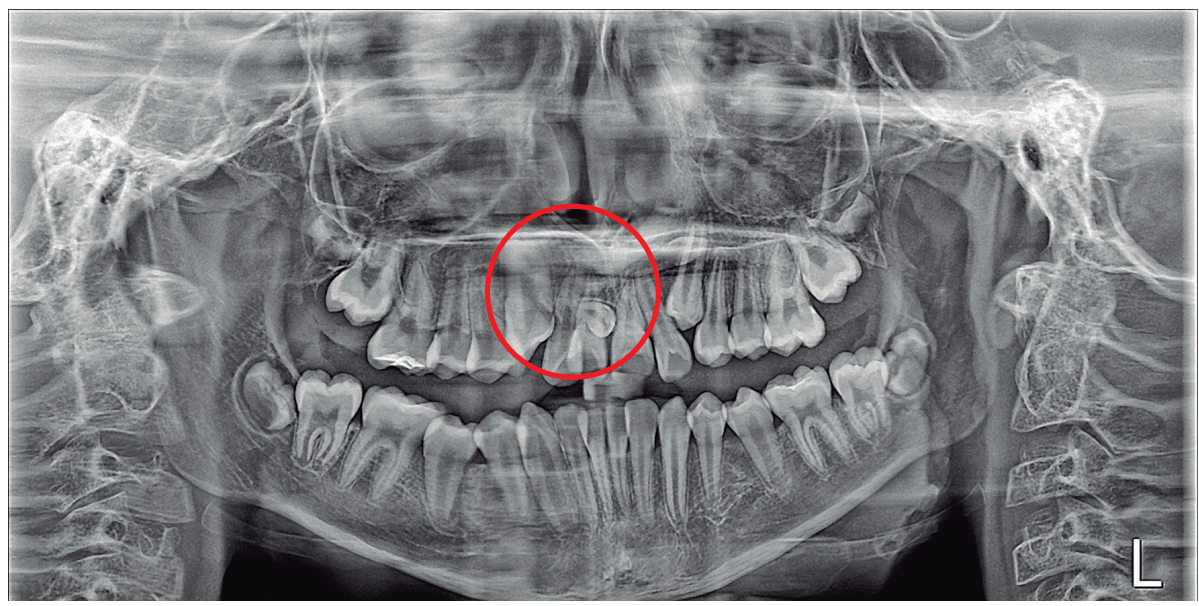

Figura 1. Presencia de diente SuPERnUMERARIO MESIOdENS DISMÓRFICO.

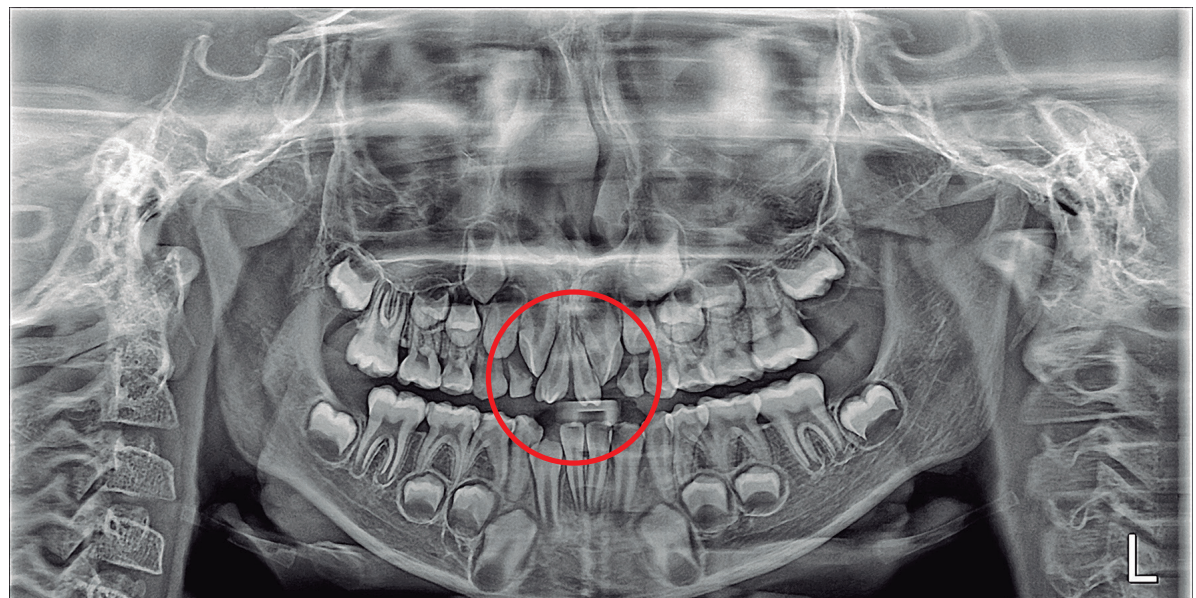

Figura 2. PRESENCIA DE DIENTE SUPERNUMERARIO MESIODENS DISMÓRFICO DUPLICADO. 
Milagros Zevallos Melgar, Carol Magaly Cárdenas-Flores,

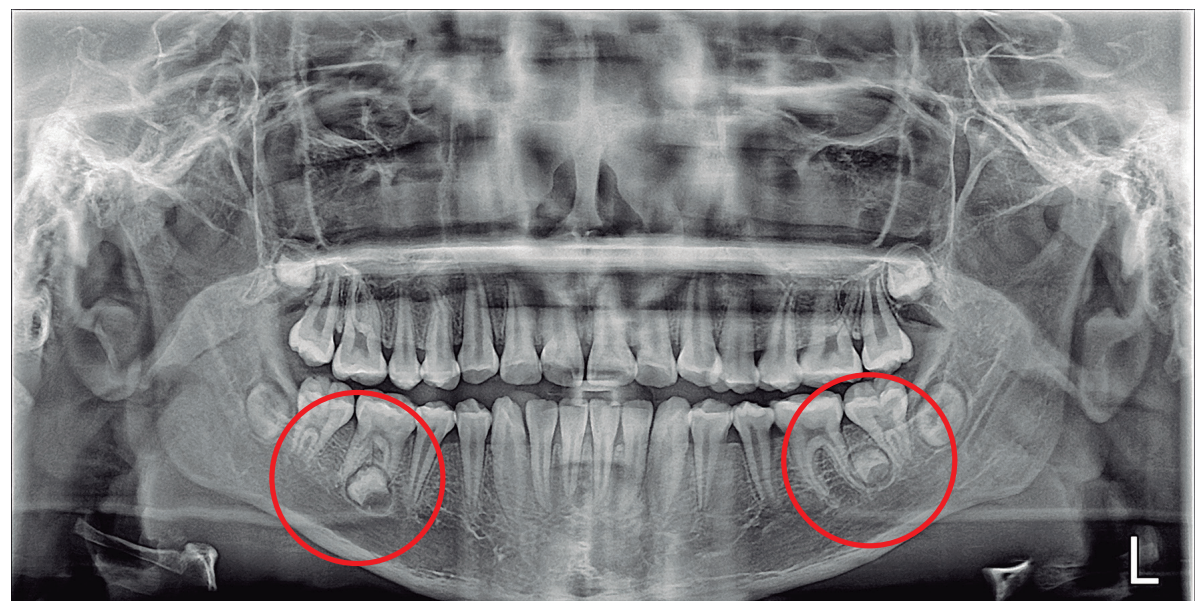

FIGURA 3. GÉRMENES DENTARIOS INFERIORES SUPERNUMERARIOS PARAPREMOLARES, DERECHA E IZQUIERDA.

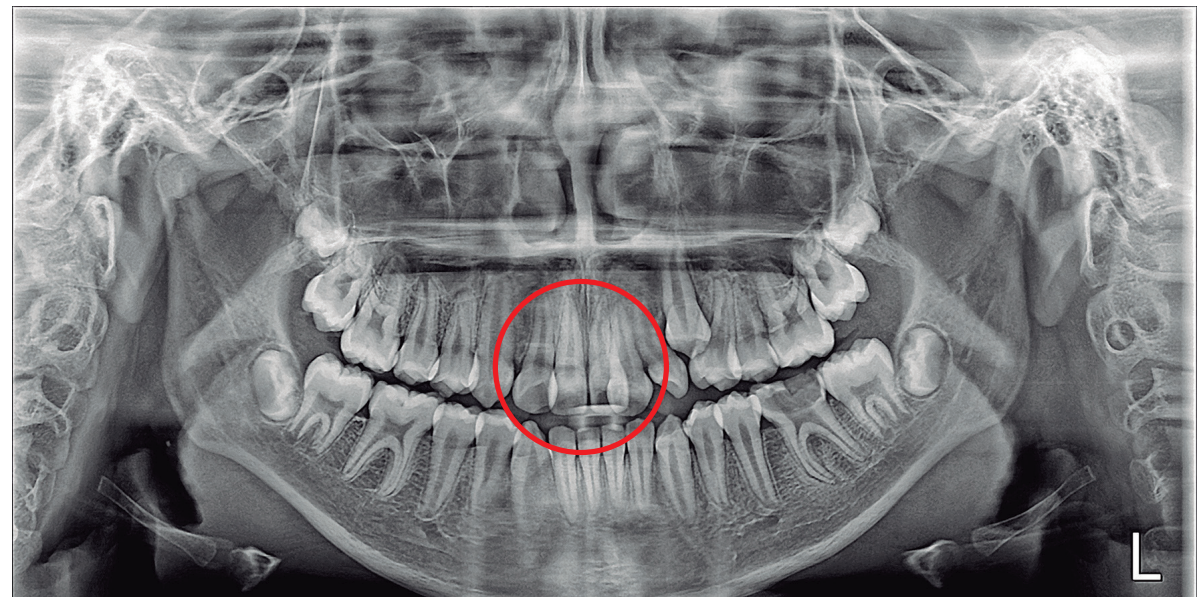

FIGURA 4. PRESENCIA DE DIENTE SUPERNUMERARIO EUMÓRFICO ENTRE INCISIVO LATERAL Y CANINO SUPERIOR. 


\section{REFERENCIAS BIBLIOGRÁFICAS}

1. Gómez de Ferraris ME, Campos Muñoz A. Embriología general humana. En: Histología y embriología bucodental. Madrid: Editorial Médica Panamericana; 1999. pp. $17-8$.

2. Scheiner MA, Sampson WJ. Supernumerary teeth: a review of the literature and four case reports. Aust Dent J. 1997; 42 (3): 160-5.

3. Calvano E, de Castro M, Rezende A. Concomitant tooth agenesis and supernumerary teeth: Report of a family. Ped. Dent J. 2009; 19 (1): 154-8.

4. Cameron A. Anomalías dentales. En: Manual de Odontología Pediátrica. 1.ra ed. Madrid: Harcourt Brace; 1998. pp. 179-220.

5. Díaz A, Orozco J, Fonseca M. Multiple hyperdontia: report of a case with 17 supernumerary teeth with non syndromic association. Med Oral Patol Oral Cir Bucal. 2009; 14: E229-E231.

6. Liu D, Zhang W, Zhang ZZ, Wu Y, Ma X. Three-dimensional evaluations of supernumerary teeth using cone-beam computed tomography for 487 cases. Oral Surg Oral Med Oral Pathol Oral Radiol Endod. 2007; 103 (3): 403-11.

7. Garvey MT, Barry HJ, Blake M. Supernumerary teeth-an overview of classification, diagnosis and management. J Can Dent Assoc. 1.ra ed. Buenos Aires: Editorial Médica Panamericana; 1992. pp. 145-67.

8. Bolaños L. Dientes supernumerarios: reporte de casos y revisión de literatura. Odontos - Int J Dent Sc. 2008; 10: 73-8.

9. Primosch RE. Anterior supernumerary teeth assessment and surgical intervention in children. Pediatric Dent. 1981; 3: 204-15.

10. Liu DG, Zhang WL, Zhang ZY, Wu YT, Ma XC. Three dimensional evaluation of supernumerary teeth using cone-beam computed tomography for 487 cases. Oral Surg Oral Med Oral Pathol Oral Radiol Endod. 2007; 103: 403-6.

11. Sánchez Bowen B. Hiperdoncia no sindrómica en dentición permanente: su distribución y frecuencia en una serie de casos de la región Metropolitana de Chile. [Tesis de grado]. Chile: Universidad de Chile; 2013.

12. Jammal N, Silva R. Prevalencia de dientes supernumerarios en una muestra mexicana. Medigraphic. 2015; 3 (2): 88-91.

13. Acosta Barreda A. Análisis radiográfico de las anomalías en el número de dientes de niños de 6 a 12 años atendidos en la especialidad de Ortodoncia de la Clínica Odontológica de la UCSM, Arequipa 2017. [Tesis de grado]. Arequipa: Universidad Católica de Santa María; 2017. 
Milagros Zevallos Melgar, Carol Magaly Cárdenas-Flores,

14. Serrano Erazo L. Frecuencia de anomalías dentarias de número en dentición mixta de pacientes atendidos en un centro de diagnóstico por imágenes de la provincia de Chiclayo, periodo 2012-2013. [Tesis de grado]. Chiclayo: Universidad Católica Santo Toribio de Mogrovejo; 2018.

CORRESPONDENCIA: Carol Magaly Cárdenas-Flores, caryto2007@ hotmail.com

ARTí́culo RECIBIDO: 04/07/2018

ARtículo ACEPTADO: 02/09/2018 